\title{
ZETA FUNCTIONS OF GROUPS: EULER PRODUCTS AND SOLUBLE GROUPS
}

\author{
MARCUS DU SAUTOY \\ Mathematical Institute, 24-29 St Giles, Oxford OX1 3LB, UK \\ (dusautoy@maths.ox.ac.uk)
}

(Received 24 April 2000)

\begin{abstract}
The well-behaved Sylow theory for soluble groups is exploited to prove an Euler product for zeta functions counting certain subgroups in pro-soluble groups. This generalizes a result of Grunewald, Segal and Smith for nilpotent groups.
\end{abstract}

Keywords: zeta function; soluble group; Euler product

AMS 2000 Mathematics subject classification: Primary 20F16; 11M99

\section{Introduction}

Let $G$ be a finitely generated group. Denote by $a_{n}^{\leqslant}(G)$ the number of subgroups of index $n$ in $G$. We can encode these numbers in a Dirichlet series which we call the zeta function of $G$ :

$$
\zeta_{G}^{\leqslant}(s)=\sum_{n=1}^{\infty} a_{n}^{\leqslant}(G) n^{-s} .
$$

We also have a zeta function encoding the lattice of normal subgroups:

$$
\zeta_{G}^{\triangleleft}(s)=\sum_{n=1}^{\infty} a_{n}^{\triangleleft}(G) n^{-s},
$$

where $a_{n}^{\triangleleft}(G)$ denotes the number of normal subgroups of index $n$ in $G$.

These functions were first introduced in $[\mathbf{3}]$ where they were studied in the particular case in which $G$ is a finitely generated torsion-free nilpotent group. These groups have the property that the global zeta function can be written as an Euler product of natural local factors defined for each prime $p$ : for $* \in\{\leqslant, \triangleleft\}$,

$$
\zeta_{G, p}^{*}(s)=\sum_{n=0}^{\infty} a_{p^{n}}^{*}(G) p^{-n s} .
$$

To say that there is an Euler product for $\zeta_{G}^{*}(s)$ in terms of these local factors is to express analytically the fact that a subgroup of finite index is the intersection in a unique way of 
subgroups of $p$-power index. For nilpotent groups this follows from the fact that a finite nilpotent group is a direct product of its Sylow $p$-subgroups.

There is another class of groups with a well-behaved Sylow theory, namely soluble groups. A number of people have asked whether this Sylow theory for soluble groups translates into an Euler product for associated zeta functions. In this paper we seek to provide an answer to this question. We prove that a pro-soluble group has a Sylow basis (see Lemma 3.2). We then show that the zeta function counting subgroups permutable with this Sylow basis has an Euler product (see Theorem 3.4).

\section{Nilpotent groups}

First we recall the proof for nilpotent groups. The most general setting is set out in the following theorem.

Theorem 2.1. Suppose that $G$ is a finitely generated group with the property that every finite quotient of $G$ is nilpotent. Then, for $* \in\{\leqslant, \triangleleft\}$,

$$
\zeta_{G}^{*}(s)=\prod_{p \text { prime }} \zeta_{G, p}^{*}(s) .
$$

Proof. We must show that if $n=p_{1}^{e_{1}} \ldots p_{k}^{e_{k}}$, where $p_{1}, \ldots, p_{k}$ are distinct primes, then

$$
a_{n}^{*}=a_{p_{1}^{e_{1}}}^{*} \ldots a_{p_{k}^{e_{k}}}^{e_{1}}
$$

(where we denote $a_{n}^{*}=a_{n}^{*}(G)$ ). Since $G$ is finitely generated, there exists a normal subgroup contained in every subgroup of index less than or equal to $n$. We may therefore suppose that $G$ is a finite nilpotent group and further that $p_{1}, \ldots, p_{k}$ are the prime divisors of $|G|$. For $i=1, \ldots, k$ let $S_{i}$ denote the (normal) Sylow $p_{i}$-complement in $G$. If $H$ is a subgroup of index $n$ in $G$, then

$$
H=\bigcap_{i=1}^{k} H S_{i}
$$

and $\left|G: H S_{i}\right|=p_{i}^{e_{i}}$. Conversely, if $H_{i}$ is a subgroup of index $p_{i}^{e_{i}}$ for each $i=1, \ldots, k$ and $H=\bigcap_{i=1}^{k} H_{i}$, then $H_{i}=H S_{i}$. Hence

$$
a_{n}^{\leqslant}=a_{p_{1}^{e_{1}}}^{\leqslant} \ldots a_{p_{k}^{e_{k}}}^{\leqslant} .
$$

(Note that if $G$ is not nilpotent, then $H$ cannot in general be written uniquely as an intersection of subgroups of prime power index.)

The subgroup $H$ is normal in $G$ if and only if $H_{i}$ is normal in $G$ for each $i=1, \ldots, k$. Hence

$$
a_{n}^{\triangleleft}=a_{p_{1}^{e_{1}}}^{\triangleleft} \ldots a_{p_{k}^{e_{k}}}^{\triangleleft} .
$$


The condition that every finite quotient of $G$ is nilpotent is equivalent to the profinite completion $\hat{G}$ of $G$ being pro-nilpotent.

For finite groups the existence of an Euler product is equivalent to the group being nilpotent.

Proposition 2.2. If $G$ is finite, then

(1) $\zeta_{G}^{\lessgtr}(s)=\prod_{p \text { prime }} \zeta_{G, p}^{\lessgtr}(s)$ if and only if $G$ is nilpotent; and

(2) $\zeta_{G}^{\triangleleft}(s)=\prod_{p \text { prime }} \zeta_{G, p}^{\triangleleft}(s)$ if and only if $G$ is nilpotent.

Proof. Let $|G|=p_{1}^{n_{1}} \ldots p_{r}^{n_{r}}$. (1) Consider the subgroups of index $|G|$. The Euler product implies that

$$
a_{p_{i}^{n_{i}}}^{\leqslant}(G)=1
$$

for each $i$. This implies that the number of Sylow $p_{i}$-subgroups is

$$
a_{|G| / p_{i}^{n_{i}}}^{\leqslant}(G)=\prod_{j \neq i} a_{p_{j}^{n_{j}}}^{\leqslant}(G)=1 .
$$

Hence there is a unique Sylow $p_{i}$-subgroup for each $i=1, \ldots, r$, which in turn implies that $G$ is nilpotent.

For (2) the same argument implies that there exists a normal Sylow $p_{i}$-subgroup for each $i=1, \ldots, r$, which again implies that $G$ is nilpotent.

However, in $[\mathbf{2}]$ we give examples of infinite non-nilpotent groups whose zeta functions enjoy an Euler product.

Theorem 2.3.

(1) The group $\mathbf{p g}=\left\langle x, y, t \mid[x, y], t^{2}=y, x^{t}=x^{-1}\right\rangle$ is not nilpotent but

$$
\zeta_{\mathbf{p g}}^{\leqslant}(s)=\zeta(s) \zeta(s-1)=\prod_{p \text { prime }} \zeta_{\mathbf{p g}, p}^{\leqslant}(s) .
$$

(2) The group $\mathbf{p m}=\left\langle x, y, m \mid[x, y], m^{2}, x^{m}=x, y^{m}=y^{-1}\right\rangle$ is not nilpotent but

$$
\zeta_{\mathbf{p m}}^{\leqslant}(s)=\left(1+2^{-s+2}\right) \zeta(s) \zeta(s-1)=\prod_{p \text { prime }} \zeta_{\mathbf{p m}, p}^{\leqslant}(s) .
$$

(3) The group $\mathbf{c m}=\left\langle x, y, t \mid[x, y], t^{2}, x^{t}=x y, y^{t}=y^{-1}\right\rangle$ is not nilpotent but

$$
\zeta_{\mathbf{c m}}^{\leqslant}(s)=\left(1+2^{-2 s+2}\right) \zeta(s) \zeta(s-1)=\prod_{p \text { prime }} \zeta_{\mathbf{c m}, p}^{\leqslant}(s) .
$$

The groups 'pg', 'cm' and 'pm' are three of the 17 wallpaper groups and are finite extensions of the free abelian group $\mathbb{Z}^{2}$ of rank 2. This Euler product is somewhat accidental. We would still be interested in providing an example to answer the following question (presumably in the negative). 
Question 2.4. If $\zeta_{G}^{\lessgtr}(s)=\prod_{p \text { prime }} \zeta_{G, p}^{\lessgtr}(s)$, then is $\hat{G}$, the profinite completion of $G$, virtually pro-nilpotent?

In contrast to the situation for subgroups, if the normal zeta function of $G$ has an Euler product, then Puchta has proved that the profinite completion of $G$ is in fact pro-nilpotent (see [4]).

Proposition 2.5. Let $G$ be a finitely generated profinite group. Then $G$ is pronilpotent if and only if $\zeta_{G}^{\triangleleft}(s)=\prod_{p \text { prime }} \zeta_{G, p}^{\triangleleft}(s)$.

\section{Soluble groups}

We can use the well-controlled Sylow structure of finite soluble groups to establish some analogue of Theorem 2.1. We recall the following definition.

Definition 3.1. Let $G$ be a finite soluble group. A set $B$ consisting of pairwise permutable Sylow $p$-subgroups of $G$, exactly one for each $p$ dividing $|G|$ together with the identity subgroup is called a Sylow basis of $G$.

Note that a finite group is soluble if and only if it has a Sylow basis.

Lemma 3.2. Let $G$ be a pro-soluble group. Then $G$ has a Sylow basis. (Here, a closed subgroup of a profinite group is said to be a $p$-Sylow group of $G$ if $P$ is a pro-p-group for which $p \nmid[G: U]$ for all open subgroups $U$ containing $P$.)

Proof. Denote the family of open normal subgroups of $G$ by $\mathcal{N}$. For each $N \in \mathcal{N}$ denote the set of all $p$-Sylow groups of $G / N$ which contain $H / N$ by $\mathcal{S}_{p}(N)$. Let $\mathcal{B}(N) \subseteq$ $\prod_{p \text { prime }} \mathcal{S}_{p}(N)$ be the set of Sylow basis of $G / N$. Since $G / N$ is a soluble finite soluble group, $\mathcal{B}(N)$ is finite and non-empty. If $M \in \mathcal{N}$ and $M \leqslant N$, then the canonical map $G / M \rightarrow G / N$ maps a Sylow basis for $G / M$ onto a Sylow basis for $G / N$. It therefore defines a canonical map of $\mathcal{B}(M)$ into $\mathcal{B}(N)$. Thus the inverse limit of the sets $\mathcal{B}(N)$ is non-empty. So there is a Sylow basis $\left\{S_{p}(N) \mid p\right.$ prime $\}$ for each $N \in \mathcal{N}$, where we interpret $S_{p}(N)=1$ for $p \nmid|G / N|$, such that if $M \leqslant N$, then $S_{p}(M)$ is mapped onto $S_{p}(N)$. The inverse limit

$$
S_{p}=\lim _{\longleftarrow} S_{p}(N)
$$

is a Sylow pro- $p$-subgroup of $G$ and our construction ensures that $S_{p} S_{q}=S_{q} S_{p}$, hence $\left\{S_{p} \mid p\right.$ prime $\}$ is a Sylow basis.

Definition 3.3. For any set of subgroups $X$ of $G$ we call a subgroup $H$ of $G X$ permutable if $S H=H S$ for all $S \in X$. We shall write $H \perp X$ in this case. If $N$ is a subgroup of $G$, we denote the set of all $X$-permutable subgroups of $N$ by $\mathcal{X}^{\perp X}(N)$. Define

$$
\zeta_{N}^{\perp X}(s)=\sum_{H \in \mathcal{X}^{\perp X}(N)}|N: H|^{-s} .
$$

When $X$ is a Sylow basis we have the following Euler product. 
Theorem 3.4. Let $G$ be a finitely generated pro-soluble group. Then, if $B$ is a Sylow basis for $G$,

$$
\zeta_{G}^{\perp B}(s)=\prod_{S_{p} \in B} \zeta_{S_{p}, p}^{\perp B}(s) .
$$

Proof. As in Theorem 2.1 we must show that

$$
a_{n}^{\perp B}(G)=a_{p_{1}^{1}}^{\perp e_{1}}\left(S_{p_{1}}\right) \ldots a_{p_{k}^{e_{k}}}^{\perp B}\left(S_{p_{k}}\right),
$$

where $n=p_{1}^{e_{1}} \ldots p_{k}^{e_{k}}$ and

$$
a_{n}^{\perp B}(N)=\operatorname{card}\left\{H \in \mathcal{X}^{\perp B}(N):|N: H|=n\right\}=\operatorname{card} \mathcal{X}_{n}^{\perp B}(N) .
$$

To prove this we may assume that $G$ is a finite soluble group and that the set $\left\{S_{p_{1}}, \ldots, S_{p_{k}}, S_{p_{k+1}}, \ldots, S_{p_{l}}\right\}$ is a Sylow basis for $G$. (Note that when $G$ is soluble we cannot in general assume that $l=k$.) We claim that the map $H \mapsto\left(H \cap S_{p_{1}}, \ldots, H \cap S_{p_{k}}\right)$ defines a bijection between $\mathcal{X}_{n}^{\perp B}(G)$ and

$$
\mathcal{X}_{p_{1}^{1}}^{\perp e_{1}}\left(S_{p_{1}}\right) \times \cdots \times \mathcal{X}_{p_{k}}^{\perp e_{k} B}\left(S_{p_{k}}\right) .
$$

First we prove that the map is well defined. The index $\left|S_{p_{i}}: H \cap S_{p_{i}}\right|=\left|S_{p_{i}} H: H\right|$ is a power of $p_{i}$ and, since $H$ is $B$-permutable, $S_{p_{i}} H$ defines a group. Hence

$$
\left|S_{p_{i}} H: H\right|=|G: H|_{p_{i}}\left|G: S_{p_{i}} H\right|_{p_{i}}^{-1}=p^{e_{i}} \cdot 1,
$$

since $S_{p_{i}} H$ contains a Sylow $p_{i}$-subgroup. This argument proves that $H \cap S_{p_{i}}$ is a Sylow $p_{i^{-}}$ subgroup of $H$ for $i=1, \ldots, k$, and the argument works equally well for $i=k+1, \ldots, l$. By the proof of Lemma 4.19 in $[\mathbf{1}]$, the Sylow subgroups $\left\{S_{p_{i}} \cap H: i=1, \ldots, l\right\}$ are pairwise permutable. Order considerations show that

$$
H=\prod_{i=1}^{l}\left(H \cap S_{p_{i}}\right) .
$$

Hence the above map is injective.

We are still required to prove that $\left(S_{p_{i}} \cap H\right) \perp B$ for $i=1, \ldots, k$. But

$$
S_{p_{i}}, \ldots, S_{p_{i}} \cap H, \ldots, S_{p_{l}}
$$

are Sylow subgroups of the group $H S_{p_{1}} \ldots S_{p_{i-1}} S_{p_{i+1}} \ldots S_{p_{l}}$ (note that this is a group, since $H \perp B$ ). Again by Lemma 4.19 in [1] ,

$$
\left\{S_{p_{1}}, \ldots,\left(S_{p_{i}} \cap H\right), \ldots, S_{p_{l}}\right\}
$$

are pairwise permutable, i.e. $\left(S_{p_{i}} \cap H\right) \perp B$.

Finally we must show that the map is surjective. Let

$$
\left(P_{1}, \ldots, P_{k}\right) \in \mathcal{X}_{p_{1}^{e_{1}}}^{\perp B}\left(S_{p_{1}}\right) \times \cdots \times \mathcal{X}_{p_{k}^{e}}^{\perp B_{k}}\left(S_{p_{k}}\right)
$$


and let $H=\bigcap_{i=1}^{k} S_{p_{1}} \ldots P_{i} \ldots S_{p_{l}}$. Since $P_{i} \perp B, H$ is a group. It is a straightforward exercise to prove that $H \perp B$ and that $H \cap S_{p_{i}}=P_{i}$. Also

$$
|G: H|=\prod_{i=1}^{k}\left|S_{p_{1}} \ldots S_{p_{l}}: S_{p_{1}} \ldots P_{i} \ldots S_{p_{l}}\right|=p_{1}^{e_{1}} \ldots p_{k}^{e_{k}}=n .
$$

Hence the map is surjective. This completes the proof of the Euler product.

In a sense this is an unsatisfactory Euler product, since the local factors $\zeta_{S_{p}, p}^{\perp B}$ are still defined globally, i.e. we count those subgroups of $S_{p}$ which commute with each element of the Sylow basis.

Since normal subgroups are $X$-permutable for any system of subgroups, one might hope that this theorem would translate into an Euler product for the zeta function counting normal subgroups in soluble groups. However, we know this is not the case, since the wallpaper groups are all soluble and their normal zeta functions do not enjoy Euler products as observed at the end of the last section. The problem in the correspondence above if we restrict to normal subgroups is that $H \mapsto\left(H \cap S_{p_{1}}, \ldots, H \cap S_{p_{k}}\right)$ does not define a surjection from $\mathcal{X}_{n}^{\triangleleft}(G)$ onto $\mathcal{X}_{p_{1}^{e_{1}}}^{\triangleleft}\left(S_{p_{1}}\right) \times \cdots \times \mathcal{X}_{p_{k}}^{\triangleleft}\left(S_{p_{k}}\right)$. All we know is that for

$$
\left(P_{1}, \ldots, P_{k}\right) \in \mathcal{X}_{p_{1}^{e_{1}}}^{\triangleleft}\left(S_{p_{1}}\right) \times \cdots \times \mathcal{X}_{p_{k}^{e_{k}}}^{\triangleleft}\left(S_{p_{k}}\right),
$$

the group $H=\bigcap_{i=1}^{k} S_{p_{1}} \ldots P_{i} \ldots S_{p_{l}}$ is $B$-permutable but not necessarily normal.

\section{References}

1. K. Doerk And T. HAWkes, Finite soluble groups, de Gruyter Expositions in Mathematics, vol. 4 (de Gruyter, Berlin, 1992).

2. M. P. F. Du Sautoy, J. J. McDermott and G. C. Smith, Zeta functions of crystallographic groups and analytic continuation, Proc. Lond. Math. Soc. 79 (1999), 511-534.

3. F. J. Grunewald, D. Segal and G. C. Smith, Subgroups of finite index in nilpotent groups, Inventiones Math. 93 (1988), 185-223.

4. J.-C. PUCHTA, Groups with multiplicative subgroup growth, Israel J. Math., in press. 